\title{
Going Beyond a Conflict of Approaches in Psychiatric Care: The Perks of Interdisciplinary Research
}

\author{
Héloïse Haliday ${ }^{1}$, Lise Demailly² \\ ${ }^{1}$ University of Burgundy, Dijon (France)| heloise.haliday@u-bourgogne.fr | \\ https://orcid.org/0000-0002-9468-3679 \\ 2 University of Lille (France) | lise.demailly@univ-lille.fr | https://orcid.org/0000-0003-4690-6652
}

\begin{abstract}
Our research studied the interactions between mental health care (MHC) professionals and users in French public psychiatry, focusing on the "tools" which professionals use in their interactions with users. We draw a typology of four main "therapeutic styles" in relation to tool use: 1 - Absence of identifiable tools. Interaction is personalized and improvised. 2- Use of rituals and habits. 3- Use of methods that have otherwise been written and formalized. 4- Use of tools, be they written or computerized, protocols guiding the interactions, or strictly standardized tools. The psychologist involved in the research $(\mathrm{HH})$ intends to show how the practice of interdisciplinary fieldwork, which implied the "trouble of having a psychologist sharing fieldwork", enabled both researchers to transform a classical methodology into a series of questions concerning the focus of data gathering and data analysis, the position and stance of both researchers, and the possibility to provide not only valid descriptions of day-to-day care practices but also correct interpretations of the unconscious feelings at stake in $\mathrm{MHC}$.
\end{abstract}

Keywords: psychiatry; sociology; clinical psychology; interdisciplinary fieldwork; care practices.

\section{Introduction}

Currently, interdisciplinarity is an inevitable framework for most researchers conducting qualitative research. However, with the need to propose interdisciplinary projects to get grants and funds comes the question of the relevance of such arranged marriages: how can they best bring their full value to research? We will argue, in this paper, that some specific areas of qualitative research, and most specifically some fields, require interdisciplinary fieldwork and data analysis to deepen the understanding of the dynamics involved in fast-changing human environments.

Psychiatric care is one of those, especially in France. For the last twenty years, French mental healthcare (MHC) professionals have been torn between two conflicting approaches: the first one, based on psychodynamic theories, pleads for a "relational" or "artisanal" (Sassolas, 2019; Venet, 2020) psychiatric practice, refusing standardized protocols and evaluation on the basis that they erase professionals' subjectivity at work. The second one, which relies on neurosciences and an evidence-based approach (Leboyer \& Llorca, 2018), argues professionals only progress and achieve best practices through the standardization of their therapeutic interactions with patients, either using scales or emental health tools. Both approaches have seen their therapeutic efficacy being scientifically validated (Hofmann \& Asnaani, 2012; Leichsenring \& Steinert, 2019). Several questions remain unanswered: do care practices only balance between psychodynamic psychotherapies and evidence-based rehabilitation sessions? Why is the conflict of approaches so acute, and the protagonists so deeply involved? How can we, as researchers, answer those questions and produce interdisciplinary scientific knowledge on the matter? And finally: what can interdisciplinarity bring to qualitative researchers studying psychiatric care?

The research we present has intended to understand MHC professionals/users' interactions in French public psychiatry through an interdisciplinary lens combining a "sociology of activity" (Bidet, 2006; Ughetto, 2018) and the psychodynamic stance. We will tell the story of why we combined the two, and how the fully interdisciplinary character of 
the research-from data gathering to data analysis and scientific writing-led us to our results, but also to a hypothesis inspired by systems psychodynamics (Fraher, 2004; Petriglieri \& Petriglieri, 2020) and psychoanalytic theory regarding the acuteness of the therapeutic battle we mentioned. Though both researchers were active all along, which is why we co-author this paper, the following developments will be written from the point of view of the psychologist. I'll use the pronoun "we" when detailing our results, then switch to the "I" to tell the story of our qualitative research process.

After describing the methodology we chose and the results we obtained, I will deal with the main methodological issue of our research: "having a psychologist sharing fieldwork" (Dasen, 2019) means a certain amount of trouble, which manifests itself in unavoidable tensions regarding four key points of any qualitative research: the positioning of researchers regarding the field itself, data gathering, data analysis, and the researchers' stance. Such "trouble", defined as the need to call apparently obvious methodological points into question, might however create interesting discussions between researchers and make the key points in any qualitative research particularly visible.

I will discuss all four elements mentioned, then try to show how my presence in the research process enabled my colleague and I to go beyond a descriptive typology of "therapeutic styles" (which was useful as a first result to disprove the simple dichotomy between psychodynamic and scientific approaches) to offer a clinical interpretation of this currently undergoing conflict in psychiatric care, based on the role and place of guilt.

\section{Research Methodology}

Our research aimed at describing clinical practice by focusing on its material aspects: we looked at work as a practical accomplishment, an activity within a specific temporality, requiring technical, epistemic, ideological, decision-making and even physical coherence, as it requires a certain use of the MHC professional's own body. Trying to describe the clinical practice of care in psychiatry however implies a series of epistemological, methodological and ethical problems (Brossard, 2013; Johansson \& Eklund, 2003; Muusse et al., 2020). Although healthcare personnel, i.e., all the members, including doctors, of multidisciplinary teams in charge of caring for individuals, readily comment on certain aspects of their practice (relations with other caregivers, use of time and space), they frequently lacked the words to describe their interactions with patients. It is therefore through the direct observation and studying of micro-situations that we've deemed it possible to reveal the professional and organizational issues in $\mathrm{MHC}$, and to understand the construction of professional cultures, their consistency, their resistance to change or their potential for evolution. We hence used an ethnographic fieldwork framework designed to help us investigate both the social reality of care, and the psychological phenomena involved (Tanggaard, 2014).

Our data gathering was partly funded by the Fédération Régionale de Recherche en santé mentale des Hauts-de-France (F2RSM, 2019-2020), and was completed by the data I collected during my own doctoral research (Haliday, 2019). We chose seven services in the Lille metropolitan area and in the Ile-de-France region, according to their theoretical and clinical care orientations, in order to constitute a relevant sample of the different practices in contemporary psychiatric care. The researcher who led the field inquiry in a given service (the fields were distributed among the two researchers according to geographical and ease of transportation parameters) was always formally introduced in a service meeting, and pre-observation interviews were scheduled with the head nurse and psychiatrist to prepare for our arrival. In each of those services, we carried ten to fifteen full days of observation, during which we conducted over 120 semi-directed interviews with professionals and users alike.

Among them, 20 were recorded and fully transcribed; the others led to extensive notetaking (recording was sometimes impossible due to material constraints, or deemed unethical). Oral consent was always obtained before recording. Signed consent forms were not used because some recordings were made directly in the field during observations. 
Though our data gathering was done before the spread of Covid-19 — which means we would probably observe many variations of our results in case we went back to the same $\mathrm{MH}$ services we visited -a mere evolution of the therapeutic styles we observed would not call into question the relevance of our typology.

In order to describe the interactions between caregivers and patients and to explain what, for professionals, takes care of them, we conducted a qualitative study based on interdisciplinary data gathering and analysis in five psychiatric sectors. The two researchers involved, a sociologist and a clinical psychologist, shared a single ethnographic methodology consisting of field observations, note-taking and semi-directed interviews with mental healthcare workers (psychologists, psychiatrists, nurses, nursing assistants...) (Beaud \& Weber, 2017). Our interviews were always conducted after observing professionals: first because people do not always do what they say they do (Wassmann \& Dasen, 1993), and second because observation and shared experiences with interviewees help dealing with "muddy" interviews (Lippke \& Tanggaard, 2014).he interviewee's thoughts and personal positions are more precise and more in depth. Secondly, in situ observation prior to the interview with a professional creates a shared reality of reference which facilitates discussion between MHC professionals and researchers.

While thematic analysis is an essential part of most qualitative research projects, and though both researchers have used microsociological or clinical thematic analysis (Paillé, 2011) in previous works, in this research there has not been any coding process per se. Thematic analysis is indeed most useful when studying social representations (Flick et al., 2015) or when dealing with clinical research interviews led by psychologists (Castarède, 2013). When studying practices, however, a choice must initially be made: will the researchers focus on what professionals think, believe, or say is happening, or will they rather investigate what is happening and ask for elicitations? In this research, we used interview excerpts as illustrations or confirmation material: first came the observations, then the interviews, which were designed to complete and deepen our understanding of "social facts" (Durkheim, 2009) as seen in the "social drama" (Hughes, 1976) that is psychiatric care work. The typology presented therefore, proceeds from a typical ethnographic iterative process, starting with observations, and followed by a gathering of explanations given by professionals themselves to verify the researchers' understanding of the scenes they observed.

In our research, focused on material frameworks, objects, tools, in other words, the "materialities" (tools, machines, technologies, spaces, etc.) that are at the heart of the very concrete individual and collective issues facing caregivers, and that engage the body and mind of each person. This choice was guided by two observations: first, that professionals needed something to help them anchor their discourse in the reality of their practice otherwise, most of them talk about what should ideally be done instead of what they currently do, only to note the latter is by no means conform to the former. When it comes to describing their day-to-day tasks, interviews with healthcare professionals can easily deviate from care itself to quality of working life issues. Asking about the tools they used to improve their therapeutic relationships with users was thus seen as a good interview starter. Second, the notion of "materiality" refers to the paradoxically invisible aspect of care work itself, in a discipline where the relationship is given pride of place, namely its inscription in concrete coordinates that partly determine the outcome. The COVID-19 epidemic provided an opportunity to grasp the importance of physical constraints in MHC work as it is done social or physical distancing, cancelling therapeutic activities and mask wearing all change care practices. In our research, we mostly focused on MHC interactions and the regulation of the professional/user interactions by the said tools: training, interview guides, e-mental health software, interaction rituals... as well as on what they do, from the point of view of the professionals, to their clinical practice.

In this context, we call a "tool" any material, symbolic or digital object that is a third party, a mediator in the care/care relationship, and whose use, as long as the tool is not standardized, leaves room for the creativity of the person who uses it. We will speak of a "protocol" to designate an explicitly formalized framework —often in stages —intended to 
guide the professional/user relationship and the interprofessional cooperation around the same patient. The formal semi-directive interviews conducted for this research were recorded with the consent of the professionals, transcribed and anonymized.

\section{Research Results}

The results of this study showed us how eclectic and heterogeneous care practices are, despite services displaying a unique, official approach. When it came to professional/user interactions, we found professionals worked with distinctive "therapeutic styles". A therapeutic style is a set of characteristics of a care interaction, be they discursive or not, oriented by a concern for therapeutic, educational and accompaniment effectiveness. Therapeutic styles are highly correlated to the absence or presence of tools, and to the nature of these tools. A service's philosophy of care is often influenced by said service's relationship to tool use (for instance, when professionals refuse using care protocols). Four styles can be distinguished, depending on the degree of formalization, by the professionals, of their interactions in MHC, and on the degree of technicality and inflexibility of the tools. These styles are not exclusive categories: they are rather complementary, for they can be seen in action in the same service at different times and places.

1. No identifiable tooling. The regulation of interaction is only of an ethical and professional nature and is based on the skills, training, professional jurisdictions or just the common sense and personality of the professionals involved. Interaction is both personalized and improvised. The practices may give rise afterwards to an exchange and collective regulation or collective reflection, but which is not formalized in methods or instructions.

2. Use of rituals. A ritual is the usual method by which a professional comes into contact with a patient. They may be specific to a particular caregiver or a group of caregivers, and can also be called routines.

3. Use of methods that have been otherwise written and formalized. These are the psychotherapeutic methods, to which some service professionals are trained: analytical psychotherapy, cognitive-behavioral therapy (CBT), psychodrama, systemic therapy, etc.

4. Use of:

a. tools (written or computerized) that encourage patient expression or self-care (Eladeb cards, various computer kits, etc.).

b. protocols guiding the interaction (possibly locally produced and revisable): interview guides, exercises created by professionals, patient assessment protocols, etc.

c. standardized tools: evaluation protocols, manuals, etc.

Relying on external tools still lets practices vary greatly, at least in cases 4-1 and 4-2, where they cannot solely be defined by the tools in use during the interaction.

It turns out that when comparing MHC practices; the researcher faces a continuum from the most improvised to the most standardized interaction, in other words to the maximum externalization of the interaction competence. In the explicit rejection of "protocolization", there is a concern that the caregiving interaction is an encounter, and it is on this condition that the interaction is considered having its own effectiveness and therapeutic value. However, in everyday care practices, the absolute protocolization of the care relationship is an unsustainable position: our results show how eclectic the practices are and how the formalization of these practices can reveal, each time, something of the caregiver's 
creativity and the subjectivity of the person being treated, in other words, a movement that is always at work "overcoming automatisms" (Stiegler, 2015) in relational work. An inventive and flexible formalization, inasmuch as it makes the tools of a team shareable with others, would be the opposite of both absolute improvisation and total standardization. It could be understood as a way for pluri professional teams to stay creative in their interactions with patients while building shared mental models (Mathieu et al.,2000) of care, and therefore be a fruitful alternative between improvisation and standardization.

\section{Discussion}

Though professionals sometimes expressed radical positions when interviewed, clinical reality always seemed more complex. The general heterogeneity of care practices in France does not exclude, though, the possibility to build a typology of therapeutic styles.

Each of the styles listed above embodies a specific vision of professionalism, a vision of organization, and a vision of what constitutes good care. In styles where formalization is strong, there is a certain distrust of the subjectivity of the caregivers (diagnostic procedures must be formalized to make them safer) and a certain distrust of training inequalities (the tool ensures a constant average quality of services). However, pendulum movements can be seen in a same service over time: after having formalized care procedures, sometimes to the point of standardization, professionals might feel the need to customize and make their tools more flexible (Morin et al. 2015). These pendulum movements certainly draw from the conscious will to attain a high quality of care, but social scientists and psychologists alike need to go beyond this sole explanation.

\subsection{Are the Research Results... enough?}

Furthermore, one could wonder, while reading, why these results matter, for they seem unsurprising: the eclecticism we observed and the consequent gap between the theoretical part of the conflict and the reality of care practices might have been predictable. What we want to stress on, in this paper, is therefore not the set of results itself, but the methodological obstacles we had to overcome to get them and the hypothesis we formulated to go deeper into the analysis of the real issue at stake, i.e., the reasons behind the acuteness of the theoretical therapeutic battle. We will try to show, in this sense, why "having a psychologist sharing fieldwork" was indeed worth the trouble.

Though researchers have already written about their "clinical perspective" on fieldwork (Schein, 1987), or stressed on the psychological aspects of being on the field, especially when dealing with the emotions arising from these experiences (Davies \& Spencer, 2010), few of them, apart from Pierre Dasen (2019), have told of the many apparently obvious aspects of research which become problematic as soon as a social scientist has to bear the "trouble of having a psychologist sharing fieldwork". Lise and I felt an inevitable tension between our disciplines regarding four key points of any qualitative research:

- the positioning of researchers regarding the field itself

- data gathering

- data analysis

- the researchers' stance

These aspects were lacking from Dasen's account of interdisciplinary fieldwork. We intend to complete the point he was making by telling the story of our research process.

\subsection{Research Positioning: In, On, or Out of the Field?}

We needed to start by our positioning in the field of MHC: I, as a psychologist, was already involved in the conflict of approaches I meant to study (on the psychodynamic side). I 
however had to conduct fieldwork the way a sociologist would, i.e., as void as possible of any preconceptions. Lise thought it was feasible because she had a specific position in the mental health field... for she had a private practice of psychoanalysis. We both agreed regarding the need to juggle between our identity-on-the-field and our identity-asresearchers. This meant we both had to deal with preconceptions: we were already involved and therefore needed the same methodology to be sure our data gathering was as objective as could be. Though we shared the same interest in psychoanalysis, and because this shared interest excluded a fully objective stance, we both had to control the influence of our preconceptions about mental health care to offer the same type of reflexive space to every professional we met. We relied on our field diaries to note our observations while in the field, but also and most importantly to record our feelings and thoughts when we got home, in a form of immediate distancing with what we had seen and heard. We also agreed several interviews had to be conducted when on the field with different professionals bearing different point of views. We decided on the questions which had to be asked to gather relevant data, to avoid biases, as much as possible, in our data collection process.

\subsection{Data Gathering: Seeing or Hearing?}

Even though we had agreed on every aspect of our data gathering method, the process itself revealed an interesting tension between observation and interviewing. Lise and I did not conduct fieldwork at the same time. The interdisciplinarity of the research -in all its richness and difficulty-was only obvious when we compared the data we gathered. We managed to bring together an intrinsically interdisciplinary material, consisting in both sociological observation and clinical psychosocial interviews.

While our research methodology was the same, our data gathering styles were slightly different. Lise expected to see things while I wanted to hear them. Lise regularly reminded me of Durkheim's perspective, saying that we should study social facts as "things" (Durkheim, 1894). She focused on observing mental health professionals-and indeed, previous researchers in sociology had demonstrated professionals, or more broadly research subjects as a whole, did not really do what they said they did (Wassmann \& Dasen, 1993). As a clinical psychologist with a psychodynamic approach, knowing people do not really do what they say just points out how important psychic life is, and how powerful "psychic reality" can be. I am more interested in what happens in the minds of individuals than by what happens in the world, for I believe what we call reality is co-constructed by individuals in relation to each other. In the constructivist perspective I work with, reality arises from a collective agreement, a shared interpretation of what happens (Audoux \& Gillet, 2011).

While Lise wanted to observe as much as she could -for the validity of the research came, in her opinion, from what she saw $-\mathrm{l}$, on the contrary, rather to focus on professional discourses, and use interviews a lot more. The classical psychoanalytic stance argues listening must prevail on sight. While a sociologist considers speech to verify his or her interpretation of what was observed, I mostly wanted to understand what was inside the professionals' minds as they worked and cared for their patients, seeking to uncover the unconscious motives behind their actions. The difference was therefore obvious in the way we treated our data. While Lise rested on her field diaries, which were full of observations and written-down bits of informal interviews, I first turned to my interview transcriptions to find meaningful excerpts. While I felt compelled to transcribe every interview as a whole, Lise transcribed just the bits she was interested in for completing her observations.

\subsection{Research Stance: Between Prescription and Description}

Our research approaches also had different goals. While Lise intended to describe reality, I intended to find ways to improve it. In this third type of difference between her stance and mine, it is around the writing of papers that we actively discussed our findings. 
In the conclusion of one of our articles, I was tempted to see formalization as a path between the total absence of formalization, the nonexistence of guidelines to help professionals navigate patient professional relationships, and the absolute standardization. Lise refused to put it that way: instead of prescribing formalization as a way to transmit and share practices in a discipline which still heavily relies on oral transmission rather than written; she saw formalization, more descriptively, as a new research result, not necessarily a way of getting rid of the conflict between care approaches.

We viewed the results of our research slightly differently. Lise saw creative formalization as our main research result, the most important thing our research had taught us: professionals were active and creative, and it turned out they still had practical margins to do their jobs the way they intended to, sometimes despite their services' official approach. I viewed creative formalization of practices as a way to appease the conflict between psychodynamic approaches, which rarely lead professionals to be in favor of formalizing because formalization is viewed as taking away the patients and the professionals subjectivities, and biomedical or cognitive approaches, which rather see formalization as a necessary prerequisite to a real scientific thinking and to evidence-based therapeutic practices.

\subsection{A Clinical Stance on Data Analysis: The Use of Interpretation}

The last point I want to make refers to what Lise and I extracted from our data. While she focused on organizing the typology, I detailed earlier in this paper; I was keener on finding correct interpretations to understand the phenomena and discourses we observed and heard of the field. We had different ways of treating the core question of our research: why was it that so many professionals were involved in such a violent and cleaving conflict between psychodynamic and cognitive-behaviorists approaches of MHC? Observing the professionals closely indeed enabled a distinction between several types of tool usage, but the main problem, which was also the reason we had started the project, was still a mystery to solve.

I started thinking maybe both sides of the conflict were fighting a single complex which they shared as a common but hidden (because unconscious) ground. The professionals I observed and interviewed always seemed to be fighting a deep feeling of powerlessness. I argue this was triggered by their patients who did not seem to get better despite all their therapeutic efforts. This powerlessness, I believe, and the correlated feeling of castration, understood as a conscious knowing of their professional limitations, created a great sense of guilt. While guilt has been studied in families of mental healthcare users (Carpentier, 2001), few research works have concentrated on the specific guilt felt by professionals (Robertson, 1994). Moreover, most new trends in mental healthcare, concentrate on transferring power from mental health professionals to users. Though this shift is needed to go towards a global recovery movement, it contributes to the repression (in the psychoanalytic sense) of guilt in mental healthcare professionals. Melanie Klein (1975) theorized guilt as the product of our hatred for the surrounding objects: feeling an urge to destroy and fearing the object's destruction altogether lead to guilt. Fundamentally, guilt is what pushes us to repair the broken, attacked, destroyed objects, and "the drive to make reparation can keep at bay the despair arising out of feelings of guilt, and then hope will prevail" (p. 342). A successful reparation process appeases the guilt; when reparation however becomes impossible - as is the case in some acute psychiatric disorders -, the guilt increases and one desperately looks for reasons to hope.

The intensity of the conflict was therefore not so much related to the personal and professional convictions that one method was really better than the other, but to the overwhelming feeling of guilt, and the correlative impossibility to tolerate frustration (i.e., therapeutic failure). It is because I heard so many professionals confess their feeling of powerlessness in front of the hospital administration, in front of the users' distress, that I could hear the conflict between psychodynamic and cognitivist approaches as being a conflict between people who could not bear-and as a psychologist, I could deeply relate to their difficulty - not having found the best way to treat their patients. They had chosen 
their side in relation to this specific clinical dilemma, and the solution to the conflict therefore did not seem to lie in choosing the right approach, but in the listening and taking care of MHC professionals' own mental health, in relation to the specificities of their clinical job and their relationships to patients.

\section{Conclusion: The Perks of Interdisciplinary Research}

Doing interdisciplinary research, in the form of ethnographic inquiry combining field observations and semi-directed interviews led by a sociologist and a psychologist, proves useful for assessing and understanding psychiatric care practices. The situation in France seemed rather absurd and symptomatic: two conflicting but equally effective approaches theoretically battling against each other to take the ascendancy. To evaluate whether psychiatric care practices were really down to two different sets of techniques, we conducted a seven-services field inquiry and drew a typology of 4 "therapeutic styles". After observing and talking to MHC professionals, the dichotomy we knew and heard about seemed altogether less rigid and less clinically valid than what we saw as a growing eclecticism/heterogeneity of MHC tools and techniques. Absolute standardization was nowhere to be observed, which made us advocate for a little "formalization" as a way of building shared mental models of care and passing professionals' know-how along.

Concluding with these results was, however, unsatisfying, both because of methodological and theoretical issues. There is a certain "trouble" of having a psychologist sharing fieldwork which should be brought to light before starting any social science research in a psychiatric setting. One's capacity to objectively and benevolently observe care practices about which one already holds several preconceptions should be discussed. The difference of focus in the data gathering process (i.e., the relative importance of seeing and hearing) can lead to an enrichment of the research or apparently incompatible data. The analysis is also a key working point: what do the researchers want to gain from data gathering, and are a psychologist's potential interpretations welcome? And, last but not least, can a social scientist working with a descriptive paradigm tolerate a psychologist's prescriptions about what should be improved in the scenes they observed and the practices they learnt about?

Furthermore, not only had it been difficult to conduct interdisciplinary fieldwork: we were still stuck with the unanswered question of the intensity of the conflict of approaches. We thus interpreted the theoretical conflict between "relational" and "scientific" approaches, i.e., between non-formalization and standardization of relations and exchanges, as a symptom of deeper emotional movements in MHC teams. The conflict which prompted us to start our research in the first place had to be seen as based on an underlying accusation from both sides: that the "others", those who chose the wrong method, were passively maintaining the patient in an institutional inertia which strongly reminds the researcher in psychiatry of the 1960s debate, in the US, about "institutionalism" (Wing, 1962). The professionals who see psychiatric care as an art of the therapeutic relationship believe standardization will suppress its very heart and soul; defenders of a more evidence-based psychiatry deem that psychodynamic approaches rely on outdated beliefs and practices and therefore contribute to therapeutic stagnation. The question of relational tools in psychiatry could thus be interpreted as a battle against what famous French psychiatrists like Jean Oury (2001), Lucien Bonnafé (1991) and so many others called alienation which is what MHC workers call "apragmatism" today, the negative symptoms associated with psychotic disorders. The result is a feeling of guilt when facing clinical inertia, which pushes every professional to choose a single "one-best-way", even if it means entrenching themselves in antagonistic positions that ignore their common therapeutic goal.

Though having to conduct fieldwork was a psychologist and for the sociologist who worked with me, quite a lot of trouble, it turns out I probably would not have formulated this specific interpretation had I not been previously observing professionals in the field. The best way for me, as a psychologist, to respect and listen to all professionals despite my own preconceptions about $\mathrm{MCH}$ and therapeutic practices, was indeed to get as close as possible to the reality of day-to-day psychiatric care. Saying psychiatry is so specific a field that interdisciplinary projects unavoidably need a researcher who knows the field from the 
inside surely would go too far. But as the interest in action research methodologies grow, and the need for clinical interventions to help and support mental healthcare teams, it seems the space for clinical fieldwork methodologies is growing. Researchers in psychology and their colleagues from other social sciences disciplines might thus have to bear a little longer-and for the better-the trouble of having to share their fieldwork experiences.

\section{References}

Audoux, C., \& Gillet, A. (2011). Recherche partenariale et co-construction de savoirs entre chercheurs et acteurs: l'épreuve de la traduction [Partenarial research and knowledge coconstruction between researchers and actors : the trial of translation]. Revue Interventions Économiques, 43, Article hal-00742885.

https://doi.org/10.4000/interventionseconomiques.1347

Beaud, S., \& Weber, F. (2017). Guide de l'enquête de terrain: produire et analyser des données ethnographiques [Field survey guide: producing and analyzing ethnographic data]. La Découverte.

Bidet, A. (2006). Sociologie du travail et activité. Le travail en actes, nouveaux regards [Sociology of work and activity. Work in action, new visions]. Octarès Editions.

Bonnafé, L. (1991). Désaliéner? folie(s) et société(s) [De-alienating ? Madness and society]. Presses universitaires du Mirail.

Brossard, B. (2013). L'organisation sociale des « hasards heureux »: Qu'est-ce qu'un soin en psychiatrie? [The social organization of serendipity: what is care in psychiatry?]. Sociologie Du Travail, 55(1), 1-19. https://doi.org/10.1016/j.soctra.2012.12.003

Carpentier, N. (2001). Le long voyage des familles : la relation entre la psychiatrie et la famille au cours du XXe siècle [The long journey of families : the relationship between psychiatry and families during the 20th century]. Sciences Sociales et Santé, 19(1), 79-106. https://doi.org/10.3406/sosan.2001.1514

Castarède, M. -F. (2013). L'entretien clinique à visée de recherche [The clinical research interview]. In C. Chiland (Ed.), L'entretien clinique (pp. 139-172). Presses universitaires de France.

Dasen, P. R. (2019). The trouble of having a psychologist sharing field work. In A. Von Poser \& A. Von Poser (Eds.), Facets of fieldwork. Essays in honor of Jürg Wassmann (pp. 255-271). Universitätsverlag Winter.

Davies, J., \& Spencer, D. (Eds.). (2010). Emotions in the Field. The Psychology and Anthropology of Fieldwork Experience. Stanford University Press.

Demailly, L., \& De la Broise, P. (2009). Les enjeux de la déprofessionnalisation [The stakes of deprofessionalisation]. Socio-Logos. Revue de l'association Française de Sociologie, 4, Article 151828371. https://doi.org/https://doi.org/10.4000/socio-logos.2305

Durkheim, É. (2009). Les règles de la méthode sociologique [The rules of sociological method]. Payot \& Rivages. (Original work published 1894)

Fraher, A. L., \& Gabriel, Y. (2014). Dreaming of flying when grounded: occupational identity and occupational fantasies of furloughed airline pilots. Journal of Management Studies, 51(6), 926-995. https://doi.org/10.1111/joms.12081

Flick, U., Foster, J., \& Caillaud, S. (2015). Researching social representations. In E. Andreouli, G. Gaskell, G. Sammut, \& J. Valsiner (Eds.), The Cambridge handbook of social representations (pp. 64-80). Cambridge University Press. https://doi.org/10.1017/CBO9781107323650.007

Haliday, H. (2019). Qui prend soin du réseau? Essai d'actualisation du paradigme de la psychothérapie institutionnelle dans le système de santé mentale français [Who cares for the network? A tentative actualization of the institutional psychotherapy paradigm in the French mental health system]. Annales Médico-Psychologiques, 177(10), 1005-1009. https://doi.org/10.1016/J.AMP.2019.09.010

Hofmann, S. G., \& Asnaani, A. (2012). The efficacy of cognitive behavioral therapy: A review of meta-analyses. Cognitive Therapy Research, 36, 427-440. https://doi.org/10.1007/s10608012-9476-1 
Hughes, E. (1976). The social drama of work. Mid-American Review of Sociology, 1(1), 1-7. http://www.jstor.org/stable/23252599

Johansson, H., \& Eklund, M. (2003). Patients' opinion on what constitutes good psychiatric care. Scandinavian Journal of Caring Sciences, 17(4), 339-346. https://doi.org/10.1046/j.02839318.2003.00233.x

Klein, M. (1975). Love, guilt and reparation and other works. MacMillan.

Leboyer, M., \& Llorca, P.-M. (2018). Psychiatrie: l'état d'urgence [Psychiatry: a state of urgency]. Fayard.

Leichsenring, F., \& Steinert, C. (2019). The efficacy of psychodynamic psychotherapy: an up-todate review. In D. Kealy \& J. S. Ogrodniczuk (Eds.), Contemporary Psychodynamic Psychotherapy (pp. 47-74). Elsevier. https://doi.org/10.1016/B978-0-12-813373-6.00004-0

Lippke, L., \& Tanggaard, L. (2014). Leaning in to "muddy" interviews. Qualitative Inquiry, 20(2), 136-143. https://doi.org/10.1177/1077800413510869

Marx, K. (2007). Les Manuscrits économico-philosophiques de 1844 [Economic and philosophic manuscripts of 1844]. Vrin.

Mathieu, J. E., Heffner, T. S., Goodwin, G. F., Salas, E., \& Cannon-Bowers, J. A. (2000). The influence of shared mental models on team process and erformance. Journal of Applied Psychology, 85(2), 273-283. https://doi.org/10.1037t/0021-9010.85.2.273

Morin, P., Bossé, P.-L., Carrier, S., Garon, S., \& Lambert, A. (2015). La personnalisation des services de santé mentale: une voie d'avenir [The personalization of mental health care services: a way forward]. Santé mentale au Québec, 40(1), 135-152. https://doi.org/https://doi.org/10.7202/1032387ar

Muusse, C., Kroon, H., Mulder, C. L., \& Pols, J. (2020). Working on and with relationships: relational work and spatial understandings of good care in community mental healthcare in trieste. Culture, Medicine and Psychiatry, 44(4), 544-564. https://doi.org/10.1007/s11013-02009672-8

Oury, J. (2001). Psychiatrie et psychothérapie institutionnelle : traces et configurations précaires [Psychiatrie and institutional psychotherapy: traces and precarious configurations]. Les éditions du champ social.

Paillé, P. (2011). Les conditions de l'analyse qualitative [Qualitative analysis conditions]. SociologieS, July 2011, 0-13. http://journals.openedition.org/sociologies/3557

Petriglieri, G., \& Petriglieri, J. L. (2020). The return of the oppressed: a systems psychodynamic approach to organization studies. Academy of Management Annals, 14(1), 411-449. https://doi.org/10.5465/annals.2017.0007.

Robertson, W. (1994). The concept of guilt. Journal of Psychosocial Nursing and Mental Health Services, 32(1), 15-18.

https://pubmed.ncbi.nlm.nih.gov/8145194/\#: :text=Guilt\%20is\%20an\%20uncomfortable\%20f eeling,the\%20uncomfortable\%20feeling\%20(distress).

Sassolas, M. (2019). Le stylo du psychiatre. Plaidoyer pour une psychiatrie relationnelle [The psychiatrist's pen. Pleading for a relational psychiatry]. ERES. https://doi.org/https://doi.org/10.3917/eres.sasso.2019.01

Schein, E. H. (1987). The Clinical Perspective in Fieldwork. Sage.

Stiegler, B. (2015). L'emploi est mort, vive le travail! Entretien avec Ariel Kyrou [Employment is dead, all hail work! Interview with Ariel Kyrou]. Editions des 1001 nuits.

Tanggaard, L. (2014). Ethnographic fieldwork in psychology: lost and found? Qualitative Inquiry, 20(2), 167-174. https://doi.org/10.1177/1077800413510876

Ughetto, P. (2018). Les nouvelles sociologies du travail. Introduction à la sociologie de l'activité [The new sociologies of work. Introduction to activity sociology]. De Boeck Supérieur.

Venet, E. (2020). Manifeste pour une psychiatrie artisanale [Manifest for an artisanal psychiatry]. Verdier.

Wassmann, J., \& Dasen, P. R. (1993). Alltagswissen. Les savoirs quotidiens. Everyday Cognition. Freiburg University. 
Wing, J. K. (1962). Institutionalism in Mental Hospitals. British Journal of Social and Clinical Psychology, 1, 38-51. https://doi.org/10.1111/j.2044-8260.1962.tb00680.x. 